\title{
Synthesis and Advanced Characterisation of Layered Platelets by Self-assembly of Long-chain Amines
}

\author{
Andrés Seral-Ascaso ${ }^{1}$, Clive Downing ${ }^{1}$, Hannah C. Nerl ${ }^{1}$, Anuj Pokle ${ }^{1}$, Sonia Metel ${ }^{1}$, Joao Coelho ${ }^{1}$, \\ John (Chuafang) Zhang ${ }^{1}$, Sang-Hoon Park ${ }^{1}$ and Valeria Nicolosi ${ }^{1}$ \\ 1. Trinity College Dublin, School of Chemistry, CRANN, AMBER \& I-Form, Dublin 2, Ireland.
}

Two-dimensional (2D) materials have emerged as promising candidates for a broad range of applications, which include energy storage[1], catalyst supports[2], transistors [3] or drug delivery agents[5].

There has been intense research to define suitable routes for the production of ultrathin nanosheets of layered materials, based either on the exfoliation of the bulk compounds by different techniques[6-9] or on the growth of the 2D materials from molecular precursors via chemical vapour deposition and wet chemistry methods. The latter is a convenient approach to obtain stable, self-standing 2D structures, due to the mild reaction conditions and the great versatility of the technique, which has been successfully applied to the synthesis of transition metal chalcogenides, layered double hydroxides or metal oxides, among others.

The synthesis of oxides and hydroxides by wet chemistry methods usually takes place within an aqueous medium, while the synthesis of chalcogenides usually requires the use of inert atmosphere and organic solvents, usually amphiphilic molecules (those composed of a non-polar hydrocarbon chain and a polar functional group, such as amine, carboxylic acid, alcohol or thiol) to avoid oxidation during synthesis.

The use of amphiphilic molecules presents several advantages due to their ability to direct or template the growth of nanostructures, since the polar functional group of the amphiphile tends to interact with the growing structure while the non-polar tails interact with each other outward.

This results in the stabilisation of the final structure, avoiding agglomeration and simplifying the further processing, besides that in some cases the amphiphilic molecules also become part of the structure, acting as a scaffold.

In this work, we explore the ability of amphiphiles (long-chain amines) to template the growth of layered, hybrid materials composed of sub-nm clusters of transition metal chalcogenides and arranged in alternate layers of clusters/amines to produce self-assembled circular platelets with diameters up to several $\mu \mathrm{m}$ and thicknesses in the range of $60-80 \mathrm{~nm}$. The synthesis of the material is performed by a wet-chemistry method involving a mixture of hexadecylamine and dodecylamine as a solvent, at relatively mild reaction conditions $\left(240^{\circ} \mathrm{C}\right.$ for $\left.1 \mathrm{~h}\right)$.

The incorporation of clusters on top of nanomaterials is a promising strategy for the development of new functionalities with fascinating potential for the development of novel applications in photonics, energy harvesting or catalysis. ${ }^{39,40}$ 
References:

[1] Wang, Q. H. et al, Nat. Nanotechnol. 7 (2012), p. 699.

[2] Cano, M. et al, Nanoscale 5 (2013), p. 10189.

[3] Radisavljevic, B. et al, Nat. Nanotechnol. 6 (2011), p. 147.

[4] Buscema, M. et al, Nano Lett. 14 (2014), p. 3347.

[5] Sun, X. M. et al, Nano Res. 1 (2008), p. 203.

[6] Hernandez, Y. et al, Nat. Nanotechnol. 3 (2008), p. 563.

[7] Coleman, J. N. et al, Science 331 (2011), p. 568.

[8] Paton, K. R. et al, Nat. Mater. 13 (2014), p. 624.

[9] Nicolosi, V. et al, Science 340 (2013), p. 1420.
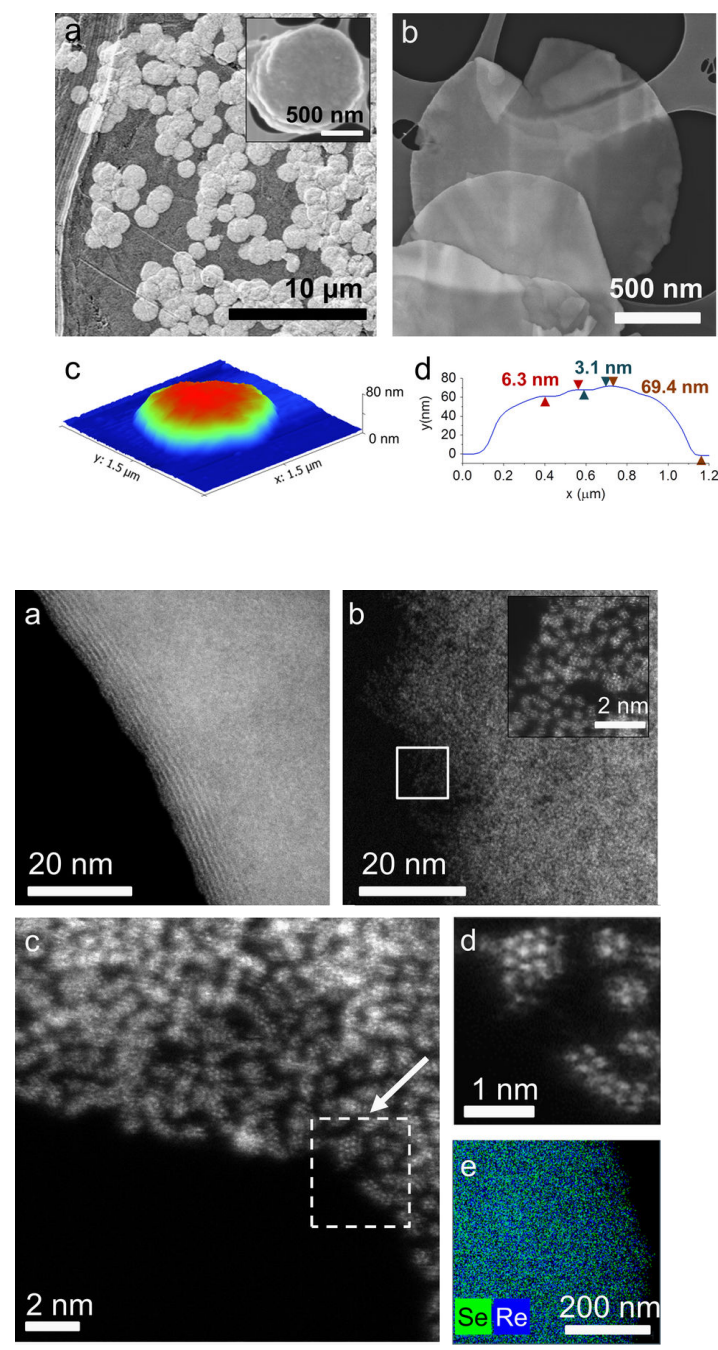

Figure 1. SEM images of platelet-like particles on top of a substrate (a) and on top of a TEM grid (b) and inset in a. AFM 3D image of a single platelet (c). The thickness profile at the centre of the platelet is displayed in $\mathrm{d}$ : the measured thickness is $69.4 \mathrm{~nm}$ and several steps are observed on top, the smallest measured step has a value of $3.1 \mathrm{~nm}$
Figure 2. High-angle annular dark-filed (HAADF) scanning transmission electron microscopy (STEM) of a region, which has a fold parallel to the electron beam confirms that the material is composed of superimposed layers (Fig. 2a), while the analysis of a flat edge reveals their granular nature (Fig. 2b). Interestingly, these granules consist of clusters of atoms with a variable morphology: most of them present a diamond (rhombus) shape, which resemble the clusters observed in ReQ2 $(\mathrm{Q}=\mathrm{S}$, $\mathrm{Se}),{ }^{41,42,43}$ while other structures, such as triangles or polyhedrons are also. 